\title{
A simple analysis of the blast initiation of detonations
}

\author{
MARTIN SICHEL $\dagger$ \\ Department of Aerospace Engineering, The University of Michigan, Ann Arbor, MI 48109, U.S.A.
}

(Received 4 March 1976; revised 21 July 1976)

\begin{abstract}
A detonation can be initiated in a fuel-oxidizer cloud by a blast wave of sufficient strength. The Zel'dovich criterion for initiation is that the blast energy must be such that when the pressure ratio across the shock front has decayed to the Chapman-Jouguet value, the distance traveled by the shock must be at least of the order of the reaction zone thickness. A modified version of this criterion has been expressed in quantitative form, and used to determine a dimensionless initiation energy using the stoichiometric value as a reference. Good agreement was found between the theory and experimentally measured initiation energies for MAPP-air, and $\mathrm{C}_{2} \mathrm{H}_{2}-\mathrm{O}_{2}$ mixtures. The theory also predicts the variation of initiation-energy with ambient pressure.
\end{abstract}

\section{Introduction}

A DETONATION can be initiated in a fuel oxidizer cloud by a blast wave of sufficient strength. The determination of the minimum or critical blast energy for initiation has been the subject of many experimental and theoretical investigations, and also is the subject of the present paper.

Instantaneous combustion at the blast front would be the simplest model to use as a basis for an initiation theory. However, in this case it can be shown that transition from blast wave to detonation behavior always occurs regardless of the blast energy (Levin, 1967; Korobeinikov, 1969). The observed existence of a critical blast energy below which no detonation occurs must therefore be tied to the ignition delay, characteristic of most combustion. This relation between the ignition delay or induction zone and the critical initiation energy was first recognized by Zel'dovitch et al. (1956), in their qualitative initiation criterion. Thus, direct initiation of spherical detonations requires that when the pressure behind the initiating blast has decayed to the Chapman-Jouguet value, the shock radius must be at least of the order of the reaction zone thickness of the mixture.

Bach et al. (1971) dealt with this problem by retaining the discontinuous combustion front; however, with a reduced heat release $Q_{e}$ which is only a fraction of the total heat release $Q$ per unit mass of mixture. The ratio $Q_{e} / Q$ was chosen as an empirical function of both the shock strength and the induction distance, i.e. the distance between the shock front and the start of combustion. This theory, although semi-empirical, appeared to predict the main phenomena which arise during blast initiation. The critical energy, however, can only be determined by the cumbersome computation of a series of shock trajectories for each set of parameters.

†P rofessor of Aerospace Engineering. 
Nicholls et al. (1974a) used an energy balance to develop a direct analytical relation for the critical initiation energy. The energy of the fluid in the induction region behind the blast front decreases as the wave decays. Nicholls et al. (1974a) argue that this decay is opposed by the energy released by combustion, and for initiation to occur the theory requires that the rate of induction zone energy decay equal the rate of combustion heat release before the blast Mach number $M_{s}$ has dropped below a critical auto-ignition value $M_{A} . M_{A}$ is a Mach number below which the Chapman-Jouguet $(C-J)$ detonations fail to propagate because the excessive growth of the induction zone causes uncoupling between the shock and combustion fronts. The choice of the energy in the induction zone essentially ignores the cumulative effect of combustion on the attenuation of the blast wave. During sample computations the theory resulted in induction zone lengths, $\Delta$, which were larger than the blast radius $R_{s}$. While there are, thus, some difficulties, the theory contains the main idea that initiation depends on a balance between blast decay and the opposing effect of combustion heat release.

Lee and Ramamurthi (1975) have introduced the concept of a critical detonation kernel to explain initiation. The integrated form of the energy equation is used to show the combined effects of blast energy release and combustion upon the Mach number $M_{s}$ of the initiating blast wave. Only the heat of combustion released between the blast center and the edge of the induction zone that is to radius $R_{s}-\Delta$ is taken into account. A critical kernel radius $R_{s}^{* *}$ is defined by equating the effects of blast energy and combustion upon $M_{s}$, the Mach number of propagation. $M_{s}^{* *}$, the Mach number corresponding to $R_{s}^{* *}$ must exceed the auto-ignition limit for initiation to occur, and this condition leads to an expression for the critical initiation energy.

Shock waves are frequently used to detonate liquid and solid explosives. Typically, the shock is applied to the explosive in the form of a pulse of a certain strength and duration, and Walker (1975), among others, has proposed a theory relating the critical energy to the shock pressure and duration. This technique differs considerably from blast ignition so that Walker's theory is not directly applicable.

The theory developed below is based on a modified form of the Zel'dovitch criterion expressed in a quantitative form. The main idea is still that the critical blast energy for initiation depends on a balance between the driving effect of the combustion energy and the induction zone growth due to the decay of the initiating blast.

\section{Formulation}

The Zel'dovitch criterion (1956) is that: for initiation the blast energy must be such that when the pressure ratio across the shock front has decayed to the Chapman-Jouguet $(C-J)$ value of the fuel oxidizer mixture, the distance traveled by the shock must be at least of the order of the reaction zone thickness of the mixture. In order to express this criterion in quantitative form the self-similar solution of Taylor (1950), and Sedov (1959) is used to describe the initiating blast. The self-similar point blast theory also was used by Lee et al. (1966) in order to compute reaction zone thickness from measured initiation energies via the Zel'dovitch criterion. 
The point blast theory does not account for the effect of combustion; therefore, the present theory can be considered as a zeroth order approximation to the initiation problem. According to the self-similar theory, the blast radius, blast Mach number, and the temperature, induced velocity and pressure immediately behind the blast can be expressed in the form:

$$
\begin{aligned}
& \frac{R_{s}}{R_{0}}=\bar{t}^{2 /(\nu+2)} \\
& M=\frac{2}{(\nu+2)} \sqrt{\left(\frac{k_{\nu}}{\alpha_{\nu}}\right) \bar{t}^{-\nu /(\nu+2)}} \\
& \frac{T_{2}}{T_{0}}=\frac{8 \gamma(\gamma-1)}{(\nu+2)^{2}(\gamma+1)^{2}} \frac{k_{\nu}}{\alpha_{\nu}} \bar{t}^{-[2 \nu(\nu+2)]} \\
& \frac{v_{2}}{c_{0}}=\frac{4}{(\nu+2)(\gamma+1)} \sqrt{\left(\frac{k_{\nu}}{\alpha_{\nu}}\right) \bar{t}^{-[\nu /(\nu+2)]}} \\
& \frac{p_{2}}{p_{0}}=\frac{8 \gamma}{(\nu+2)(\gamma+1)} \frac{k_{\nu}}{\alpha_{\nu}} \bar{t}^{-[2 \nu /(\nu+2)]} .
\end{aligned}
$$

Here the subscript zero refers to the undisturbed medium and subscript two refers to conditions immediately downstream of the shock. The dimensionless time $\bar{t}$ is defined by

$$
\bar{t}=t / \tau_{0} ; \quad \tau_{0}=R_{0} / C_{0} \sqrt{ }\left(k_{\nu} / \alpha_{v}\right)
$$

and $R_{0}$, the characteristic explosion radius, is related to the blast energy $E$ by

$$
R_{0}=\left(E / k_{\nu} \rho_{0} C_{0}^{2}\right)^{1 / \nu} .
$$

The geometric factors $\nu$ and $k_{\nu}$ are 1,2,3 and $1,2 \pi, 4 \pi$, respectively for plane, cylindrical and spherical waves. The parameter $\alpha_{\nu}$, which is given in Table 1, depends on both $\nu$ and the ratio of specific heats $\gamma . \rho_{0}$ and $C_{0}$ are the density, and the speed of sound in the undisturbed fuel-oxidizer mixture.

The blast decay is essentially complete by the time $R_{s} \sim R_{0}$ or $t \sim 0\left(\tau_{0}\right)$ where $\tau_{0}$ can be considered a characteristic explosion time. The strong blast theory fails

Table 1. Variation of the blast wave parameter $\alpha_{\nu}$ with the ratio of specific heats, $\gamma$

\begin{tabular}{cccc}
\hline$\gamma$ & $\nu=1.0$ & $\nu=2.0$ & $\nu=3.0$ \\
\hline 1.1 & 2.9383 & 2.6449 & 2.2997 \\
1.2 & 1.9114 & 1.7622 & 1.5383 \\
1.3 & 1.3620 & 1.2661 & 1.1005 \\
1.4 & 1.0264 & 0.9666 & 0.8428 \\
1.5 & 0.8163 & 0.7711 & 0.6743 \\
1.6 & 0.6695 & 0.6346 & 0.5572 \\
1.7 & 0.5619 & 0.5356 & 0.4716 \\
\hline
\end{tabular}


before this point is reached; however, the phenomena relevant to initiation occur at much smaller values of $R_{s}$.

The Zel'dovitch criterion is now modified by applying it at the critical blast radius $R_{s}^{*}$, defined by Korobeinkov et al. (1972) as that radius where the blast energy and the energy of combustion inside the blast front are equal. The heat released by combustion will begin to significantly influence propagation at this radius, and the transition from blast wave to $\mathrm{C}-\mathrm{J}$ behavior, if it occurs, takes place near the critical radius (Bach et al., 1971; Levin, 1967). Using an asterisk to denote blast wave properties at the critical radius, the modified Zel'dovitch criterion becomes

$$
\frac{\Delta^{*}}{R_{s}^{*}} \leq \delta
$$

$\Delta^{*}$ is the reaction length and the parameter $\delta$, which must be less than one, depends on the details of the initiation process. The equality holds in (8) when the blast energy equals the critical value $E_{c}$.

By combining eqns (1), (2), (7) and (8), it is now readily shown that

$$
E_{c}=\rho_{0} C_{0}^{2} M^{* 2}\left(\frac{\Delta^{*}}{\delta}\right)^{\nu} \alpha_{\nu}\left(\frac{\nu+2}{2}\right)^{2}
$$

and (9) provides a quantitative statement of the Zel'dovitch criterion. The difficulty is that the parameter $\delta$ depends on the coupling between combustion and the blast front for which a detailed analysis is not yet available. The critical initiation energy will vary with the fuel oxidizer mixture ratio. If the critical energy is normalized using the stoichiometric value $E_{c s}$ as reference the normalized critical initiation energy

$$
\frac{E_{c}}{E_{c s}}=\left(\frac{M^{*}}{M_{s}^{*}}\right)^{2}\left(\frac{\Delta^{*}}{\Delta_{*}^{*}}\right)^{\nu}\left(\frac{\rho_{0} C_{0}^{2} \alpha_{\nu}}{\rho_{o s} C_{o s}^{2} \alpha_{v s}}\right)
$$

will be independent of $\delta$ provided that this parameter does not vary appreciably with the mixture ratio. Stoichiometric quantities are denoted by the subscript $s$.

The normalized initiation criterion (10) has the advantage that it does not depend on $\delta$, and hence on a detailed consideration of combustion-blast front coupling. If the critical energy of initiation is known for any one mixture ratio, (10) can be used to determine $E_{c}$ for any other fuel-oxidizer mixture. First, however, (10) must be expressed in terms of parameters related to the propagation properties of $\mathrm{C}-\mathrm{J}$ detonations and to the kinetics of the combustion reaction. For this purpose it is convenient to express $M^{*}$ in terms of the dimensionless time $\bar{t}^{*}$ using (2). At the critical blast radius the blast energy $E$ equals the combustion energy contained inside the radius $R_{s}^{*}$. From this condition and (1) it follows that

$$
\bar{t}^{*}=\left(\nu C_{0}^{2} / Q\right)^{(\nu+2) / 2 \nu}
$$


where $Q$ is the heat of combustion per unit mass of fuel oxidizer mixture. $\bar{t}^{*}$ can be related to the properties of a $\mathrm{C}-\mathrm{J}$ detonation propagating through the mixture under consideration if it is assumed that $Q$ is constant and equal to the $\mathrm{C}-\mathrm{J}$ value. For most C-J detonations $M_{c}^{2} \gg 1$, where $M_{c}$ is the Mach number of propagation, and then it can be shown (Nicholls et al., 1974b) that $Q$ is related to $M_{c}$ by

$$
Q \cong \frac{C_{0}^{2} M_{c}^{2}}{2\left(\gamma_{2}^{2}-1\right)}
$$

where $\gamma_{2}$ is the ratio of specific heats of the products of combustion. Equation (12) is derived by assuming that the fluid behaves as a perfect gas both upstream and downstream of the detonation. Then, combining (11) and (12) results in

$$
\bar{t}^{*}=\left[\frac{2 \nu\left(\gamma_{2}^{2}-1\right)}{M_{c}^{2}}\right]^{(\nu+2) / 2 \nu}
$$

the desired relation between $\bar{t}^{*}$ and C-J properties.

Immediately behind the leading shock the fluid moves with velocity $\left(\dot{R}_{s}-v_{2}\right)$ relative to the shock front. If $\tau_{1}$ is an induction or characteristic reaction time, the induction distance $\Delta$ can be approximated by

$$
\Delta=\tau_{1}\left(\dot{R}_{s}-v_{2}\right)=\tau_{1} C_{0} M(\gamma-1) /(\gamma+1) .
$$

The relative velocity, and $\tau_{1}$, will change as the fluid moves away from the shock; however, in the early stages of blast initiation, while the temperature $T_{2}$ behind the shock is high, $\tau_{1}$ and hence $\Delta / R_{s}$ will be small so that (14) should be a reasonable approximation. Now combining (2) and (14) with (10) results in

$$
\frac{E_{c}}{E_{c s}}=\eta\left(\overline{t_{s}^{*}} \frac{\tau_{1}^{*}}{\overline{t^{*}}}\right)_{1, s}^{v}
$$

with

$$
\eta=\left[\frac{C_{0}}{C_{0 s}}\left(\frac{\gamma-1}{\gamma+1}\right)\left(\frac{\gamma_{s}+1}{\gamma_{s}-1}\right)\right]^{\nu}\left(\frac{\alpha_{\nu s}}{\alpha_{\nu}}\right)^{(1 / 2)(\nu+2)}
$$

Equation (15) provides a relation for the normalized initiation energy in terms of $\bar{t}^{*}$, which is related to C-J properties by (13), and $\tau_{1}^{*}$, which is governed by the kinetics of the combustion reaction.

A kinetic model for the induction chemistry or an empirical formula is needed to evaluate the induction time $\tau_{1}$. The simplest kinetic scheme which takes the influence of ambient density $\rho_{0}$ into account is the second order irreversible reaction

$$
A+B \stackrel{k_{f}}{\longrightarrow} C
$$


where $A$ is the fuel, $B$ the oxidizer, $C$ the combustion products, and $k_{f}$ the forward rate constant. This model has been adopted here. With $\left(A_{0}\right)$ and $(B)_{0}$ the initial concentrations of fuel and oxidizer, a characteristic reaction time $\tau_{1}$ can be defined by

$$
\tau_{1}=\left|(A)_{0} /[\mathrm{d}(A) / \mathrm{d} t]_{0}\right|=1 / k_{f}(B)_{0}
$$

Now, assuming that $k_{f}$ follows an Arrhenius law so that

$$
k_{f}=(1 / \kappa) \exp \left(-E_{1} / \mathscr{R T}\right)
$$

$\tau_{\mathrm{l}}$ will be given by

$$
\tau_{1}=\left[\kappa /(B)_{0}\right] \exp \left(E_{1} / \mathscr{R} T\right)
$$

Since many of the induction reactions in combustion are second order, the model (16), though extremely simple, is not unreasonable. A number of empirical formulas for the induction time have the form (Schott and Kinsey, 1958; White, 1967)

$$
\ln \left[(A)^{m}(B)^{1-m} \tau_{1}\right]=A+B / T
$$

which agrees with (19).

When the oxidizer is a mixture of nitrogen and oxygen, $(B)_{0}$, the initial concentration of oxygen behind the shock is given by

$$
(B)_{0}=\left(\frac{\gamma+1}{\gamma-1}\right) \rho_{0}\left[32\left(1+r_{\mathrm{N}}\right)\left(\nu_{s} \phi+1\right)\right]^{-1}
$$

with $r_{\mathrm{N}}$ the nitrogen oxygen ratio by mass, $\nu_{s}$ the stoichiometric fuel-oxidizer mass ratio and $\phi$ the equivalence ratio. $\nu_{s} \phi$ is the actual fuel-oxidizer mass ratio of the mixture.

Taking $T$ in (19) as $T_{2}$, the temperature immediately downstream of the shock, and combining (3), (15), (19) and (21) yields the following expression for the normalized initiation energy:

$E_{c} / E_{c s}=\eta\left\{\left(\bar{t}_{s}^{*} / \bar{t}^{*}\right)\left[\left(1+\nu_{s} \phi\right) /\left(1+\nu_{s}\right)\right]\right\}^{\nu} \exp \nu B_{\nu}\left(E_{1} / \mathscr{R} T_{0}\right)\left(\bar{t}^{* 2 \nu /(\nu+2)}-\bar{t}_{s}^{* 2 \nu /(\nu+2)}\right)$

where

$$
B_{\nu}=\left[(\nu+2)^{2}(\gamma+1)^{2} / 8 \gamma(\gamma-1)\right]\left(\alpha_{\nu} / k_{\nu}\right)
$$

The direct expression (9) for the critical initiation energy $E_{c}$ also can be related to the kinetic and C-J parameters using (2), (14) and (19) so that

$$
E_{c}=\rho_{0}^{1-\nu} C_{0}{ }^{\nu+2} \bar{t}^{* \nu \nu} \delta^{-\nu} D_{\nu} \exp \left(\frac{\nu B_{0} E_{1}}{\mathscr{R} T_{0}} \bar{t}^{* 2 \nu /(\nu+2)}\right)
$$


with

$$
D_{\nu}=\left[32 \kappa\left(1+r_{\mathrm{N}}\right)\left(\nu_{s} \phi+1\right)\left(\frac{2}{\nu+2}\right)\right]^{\nu}\left(\frac{\gamma-1}{\nu+1}\right)^{2 \nu} \alpha_{\nu}\left(\frac{k_{\nu}}{\alpha_{\nu}}\right)^{(1 / 2)(\nu+2)}
$$

Equation (23) shows how the critical initiation energy $E_{c}$ varies with the ambient density $\rho_{0}$. Thus, assuming that the C-J Mach number $M_{c}$, and the parameter $\delta$ are independent of $\rho_{0}$, it follows that $E_{c} \infty \rho_{0}{ }^{1-\nu}$.

\section{Comparison with experiment}

Critical initiation energies for MAPP gas-air mixtures have been measured by Collins and Parsons (1973) and by Fry and Nicholls (1974). Lee and Matsui (1976) have measured initiation energies in acetylene oxygen mixtures.

The Collins and Parsons (1973) experiments were made by placing an explosive charge at one end of a plastic bag $4 \times 4 \times 20 \mathrm{ft}$ long. Detonation was observed before any appreciable interaction with the walls of the bag could occur; hence, these tests corresponding closely to initiation by a point charge with $\nu=3$. The Fry-Nicholls (1974) experiments were made in a pie-shaped shock tube designed to simulate cylindrical clouds. This shock tube has an included angle of $20^{\circ}$ and is 2.05 in. high. The initiating charge was placed at the vertex of the pie-shaped tube, and these tests thus corresponded closely to cylindrical symmetry with $\nu=2$. The Collins-Parsons (1973) data, which has been taken from the plot of critical threshold energy vs \% MAPP in air by volume, is reproduced in Table 2 below. The Fry-Nicholls (1974) data, reproduced from Nicholls et al. (1973) is shown in Table 3. In each case, $E_{c} / E_{c s}$ was computed from the data and tabulated as a function of the \% MAPP by volume of the fuel-air mixture.

Lee and Matsui (1976) used an electric spark discharge to initiate cylindrical detonations in a chamber $13 \mathrm{~cm}$ in dia. and $3.7 \mathrm{~cm}$ wide. Only the spark energy released during the first quarter cycle of the discharge is effective in producing initiation, and this energy is reported as the critical initiation energy in Table 4. Here $E_{c} / E_{c s}$ is also tabulated as a function of the \% $\mathrm{C}_{2} \mathrm{H}_{2}$ by volume of the $\mathrm{C}_{2} \mathrm{H}_{2}-\mathrm{O}_{2}$ mixture.

Table 2. Critical initiation energy MAPP-air mixtures (Collins-Parsons (1973) bag tests)

\begin{tabular}{ccccr}
\hline $\begin{array}{c}\text { \%MAPP } \\
\text { by vol. }\end{array}$ & $\begin{array}{c}\text { \%MAPP } \\
\text { by wt. }\end{array}$ & $\begin{array}{c}\text { Equivalence } \\
\text { ratio }(\phi)\end{array}$ & $\begin{array}{c}E_{c} \\
(\mathrm{~J})\end{array}$ & \multicolumn{1}{c}{$\frac{E_{c}}{E c_{s}}$} \\
\hline 3.48 & 5.12 & 0.777 & $2.25 \times 10^{6}$ & 25.40 \\
3.65 & 5.36 & 0.816 & $3.44 \times 10^{5}$ & 3.89 \\
4.25 & 6.23 & 0.957 & $8.38 \times 10^{4}$ & 0.95 \\
5.00 & 7.30 & 1.134 & $1.01 \times 10^{5}$ & 1.14 \\
6.93 & 10.03 & 1.605 & $1.80 \times 10^{5}$ & 2.04 \\
8.25 & 11.86 & 1.938 & $4.40 \times 10^{5}$ & 4.98 \\
9.45 & 13.51 & 2.249 & $9.39 \times 10^{5}$ & 10.62 \\
9.85 & 14.06 & 2.355 & $2.25 \times 10^{5}$ & 25.40 \\
\hline
\end{tabular}


Table 3. Critical initiation energy MAPP-air mixtures (Fry-Nicholls (1974) shock tube tests)

\begin{tabular}{|c|c|c|c|c|}
\hline $\begin{array}{c}\text { \% MAPP } \\
\text { by vol. }\end{array}$ & $\begin{array}{c}\text { \% MAPP } \\
\text { by wt. }\end{array}$ & $\begin{array}{c}\text { Equivalence } \\
\text { ratio }(\phi)\end{array}$ & $\begin{array}{c}E_{c} \\
(\mathrm{~J} / \mathrm{cm})\end{array}$ & $\frac{E_{c}}{E_{c s}}$ \\
\hline 2.9 & 4.28 & 0.643 & 19,490 & 6.086 \\
\hline 3.3 & 4.86 & 0.736 & 9,552 & 2.980 \\
\hline 3.8 & 5.58 & 0.851 & 5,359 & 1.672 \\
\hline 4.3 & 6.30 & 0.968 & 3,035 & 0.947 \\
\hline 6.0 & 8.72 & 1.376 & 3,716 & 1.159 \\
\hline 6.8 & 9.85 & 1.572 & 4,740 & 1.479 \\
\hline 8.5 & 12.21 & 2.002 & 10,506 & 3.278 \\
\hline 9.7 & 13.85 & 2.315 & 18,178 & 5.663 \\
\hline
\end{tabular}

Table 4. Critical initiation energy acetylene-oxygen mixtures, $p_{0}=$ ambient pressure $=100$ torr (from Lee-Matsui (1976) spark discharge tests)

\begin{tabular}{|c|c|c|c|c|}
\hline $\begin{array}{l}\% \mathrm{C}_{2} \mathrm{H}_{2} \\
\text { by vol. }\end{array}$ & $\begin{array}{l}\% \mathrm{C}_{2} \mathrm{H}_{2} \\
\text { by wt. }\end{array}$ & $\begin{array}{l}\text { Equivalence } \\
\text { ratio }(\phi)\end{array}$ & $\begin{array}{c}E_{\mathrm{c}} \\
(\mathrm{J} / \mathrm{cm})\end{array}$ & $\frac{E_{\mathrm{c}}}{E_{c s}}$ \\
\hline 20.0 & 16.88 & 0.625 & $3.6 \times 10^{-1}$ & 3.08 \\
\hline 28.6 & 24.55 & 1.000 & $1.17 \times 10^{-1}$ & 1.00 \\
\hline 40.0 & 35.14 & 1.667 & $8.4 \times 10^{-2}$ & 0.718 \\
\hline 50.0 & 44.83 & 2.500 & $8.4 \times 10^{-2}$ & 0.718 \\
\hline 60.0 & 54.93 & 3.750 & 2.5 & 21.4 \\
\hline
\end{tabular}

Table 5. Critical initiation energy, theoretical results for MAPP-air mixtures

\begin{tabular}{|c|c|c|c|c|c|c|c|}
\hline \multirow{2}{*}{$\begin{array}{l}\text { Equivalence } \\
\text { ratio }(\phi)\end{array}$} & \multirow{2}{*}{$\begin{array}{l}\text { \% MAPP† } \\
\text { by vol. }\end{array}$} & \multirow[b]{2}{*}{$\boldsymbol{M}_{\mathrm{c}}$} & \multirow[b]{2}{*}{$\gamma_{2}$} & \multicolumn{2}{|c|}{$\begin{array}{c}\text { Cylindrical wave } \ddagger \\
\qquad \nu=2\end{array}$} & \multicolumn{2}{|c|}{$\begin{array}{c}\text { Spherical wave } \\
\qquad \nu=3\end{array}$} \\
\hline & & & & $\bar{t}^{*}$ & $E_{c} / E_{c s}$ & $\bar{t}^{*}$ & $E_{c} / E_{c s}$ \\
\hline 0.400 & 1.831 & 4.13 & 1.2661 & $1.41 \times 10^{-1}$ & 73.9 & 0.275 & 133.1 \\
\hline 0.600 & 2.722 & 4.71 & 1.2218 & $8.89 \times 10^{-2}$ & 4.61 & 0.186 & 14.53 \\
\hline 0.660 & 2.984 & 4.84 & 1.2068 & $7.78 \times 10^{-2}$ & 2.76 & 0.167 & 6.14 \\
\hline 0.888 & 3.974 & 5.20 & 1.7668 & $5.34 \times 10^{-2}$ & 1.070 & 0.122 & 1.164 \\
\hline 1.000 & 4.456 & 5.30 & 1.1651 & $5.09 \times 10^{-2}$ & 1.000 & 0.117 & 1.000 \\
\hline 1.122 & 4.974 & 5.41 & 1.1632 & $4.83 \times 10^{-2}$ & 0.950 & 0.112 & 0.896 \\
\hline 1.361 & 5.969 & 5.51 & 1.1868 & $5.38 \times 10^{-2}$ & 1.152 & 0.123 & 1.305 \\
\hline 1.605 & 6.964 & 5.53 & 1.2196 & $6.37 \times 10^{-2}$ & 1.713 & 0.141 & 2.64 \\
\hline 1.854 & 7.951 & 5.50 & 1.2434 & $7.21 \times 10^{-2}$ & 2.50 & 0.157 & 5.02 \\
\hline 2.109 & 8.955 & 5.46 & 1.2603 & $7.91 \times 10^{-2}$ & 3.52 & 0.169 & 8.95 \\
\hline
\end{tabular}

†MAPP composition: methyl acetylene $\left(\mathrm{C}_{3} \mathrm{H}_{4}\right), 35.91 \%$; propadiene $\left(\mathrm{C}_{3} \mathrm{H}_{4}\right), 24.48 \%$; propane $\left(\mathrm{C}_{3} \mathrm{H}_{8}\right), 18.58 \%$; $\left(\mathrm{C}_{4} \mathrm{H}_{10}\right), 13.74 \%$; propylene $\left(\mathrm{C}_{3} \mathrm{H}_{6}\right), 7.29 \%$. Molecular wt. $=43.37$. Stoichiometric mixture: $4.434 \%$ by vol.; $\nu_{s}=$ stoich. fuel-air mass ratio $=0.06946$.

†Other parameters used in the theory: $\gamma=1.4 ; \alpha_{2}=0.9666 ; B_{2}=3.165 ; T_{0}=298.16^{\circ} \mathrm{K}$; $\alpha_{3}=0.8428 ; B_{3}=2.156$. 
Equation (22) was used to compute $E_{c} / E_{c s}$ from the theory developed above. The results of the theoretical calculations are shown in Table 5 for MAPP-air, and in Table 6 for $\mathrm{C}_{2} \mathrm{H}_{2}$-oxygen. $M_{c}$ and $\gamma_{2}$ which are needed to compute $\bar{t}^{*}$ from (13) were obtained using the Gordon-McBride Program (1971) for detonation properties.

The author is not aware of measurements of activation energies for the induction reaction in MAPP-air mixtures. Hence $E_{1} / \mathscr{R}$ for MAPP-air was determined, here, by requiring the computed curve of $E_{c} / E_{c s}$ vs \% MAPP and the curve drawn using the Fry-Nicholls (1974) data to match at one point. In this case, the point where $E_{c} / E_{c s}=2.5$ and the mixture contains $7.95 \%$ MAPP by volume was arbitrarily chosen. Then $E_{1} / \mathscr{R}$ was found to have the value of $3350^{\circ} \mathrm{K}$. This value does not appear unreasonable although considerably lower than the values of 8700 and $9130^{\circ} \mathrm{K}$ reported for acetylene oxygen (White, 1967) and diluted hydrogen oxygen mixtures (Schott and Kinsey, 1958), respectively. In the case of MAPP-air the variation of $\gamma$, the ratio of specific heats of the unburned mixture is very small; hence, it was possible to take $\eta \cong 1$ and to use constant values for $B_{\nu}$ and $\alpha_{\nu}$ in the calculations.

There are small disparities between the MAPP compositions quoted by Collins and Parsons (1973), Fry and Nicholls (1974) and used here. The quoted compositions and the mixture properties are summarized in Table 7 , and it can be seen that the differences in molecular weight and stoichiometric mixture composition are small. The composition and MAPP C-J properties used here were taken from Sichel and $\mathrm{Hu}$ (1973). The measured and theoretical values of $\left(E_{c} / E_{c s}\right)$ are all plotted vs the \% MAPP in air by volume without adjustment for the slight differences in composition for, as Table 7 indicates, these differences should not have large effects on the results.

The experimental and theoretical values of $E_{c} / E_{c s}$ for MAPP-air are compared in Fig. 1. The theory and experiment are in remarkable agreement. The theory appears to confirm that the difference between the Collins-P arsons (1973) and Fry-Nicholls (1974) results are mainly due to the different geometries of the test apparatus used,i.e. $\nu=2$ for the Fry-Nicholls (1974) tests and $\nu=3$ for the Collins-P arsons (1973) tests. The theory also appears to predict detonation limits reasonably well and reproduces the skewness of the $E_{c} / E_{c s}$ curves relative to the stoichiometric mixture composition. Agreement appears to be best for rich mixtures; for lean mixtures the theory underestimates $E_{c} / E_{c s}$.

From Table 6 it is evident that the variation of $\gamma$ is appreciable in the case of $\mathrm{C}_{2} \mathrm{H}_{2}-\mathrm{O}_{2}$ so that variations in $\eta, \alpha_{\nu}$ and $B_{v}$ must be taken into account in the computations. Soot formation becomes appreciable when the proportion of $\mathrm{C}_{2} \mathrm{H}_{2}$ exceeds $50 \%$ by volume, and then eqn (13) for $\bar{t}^{*}$ will no longer be valid. Hence, theoretical calculations were only made for mixtures up to $50 \% \mathrm{C}_{2} \mathrm{H}_{2}$. Computations were made for $E_{1} / \mathscr{R}=8700$ and $13,880^{\circ} \mathrm{K}$. The first value corresponds to that reported by White (1967) for very lean $\mathrm{C}_{2} \mathrm{H}_{2}-\mathrm{O}_{2}$ mixtures; the second value was determined by equating theoretical and experimental values of $E_{c} / E_{c s}$ for the $20 \% \mathrm{C}_{2} \mathrm{H}_{2}-\mathrm{O}_{2}$ mixture.

Theory and experiment for $\mathrm{C}_{2} \mathrm{H}_{2}-\mathrm{O}_{2}$ are compared in Fig. 2, and are in good agreement. The theory reproduces the skewness of the $E_{c} / E_{c s}$ curve with respect 
M. Sichel
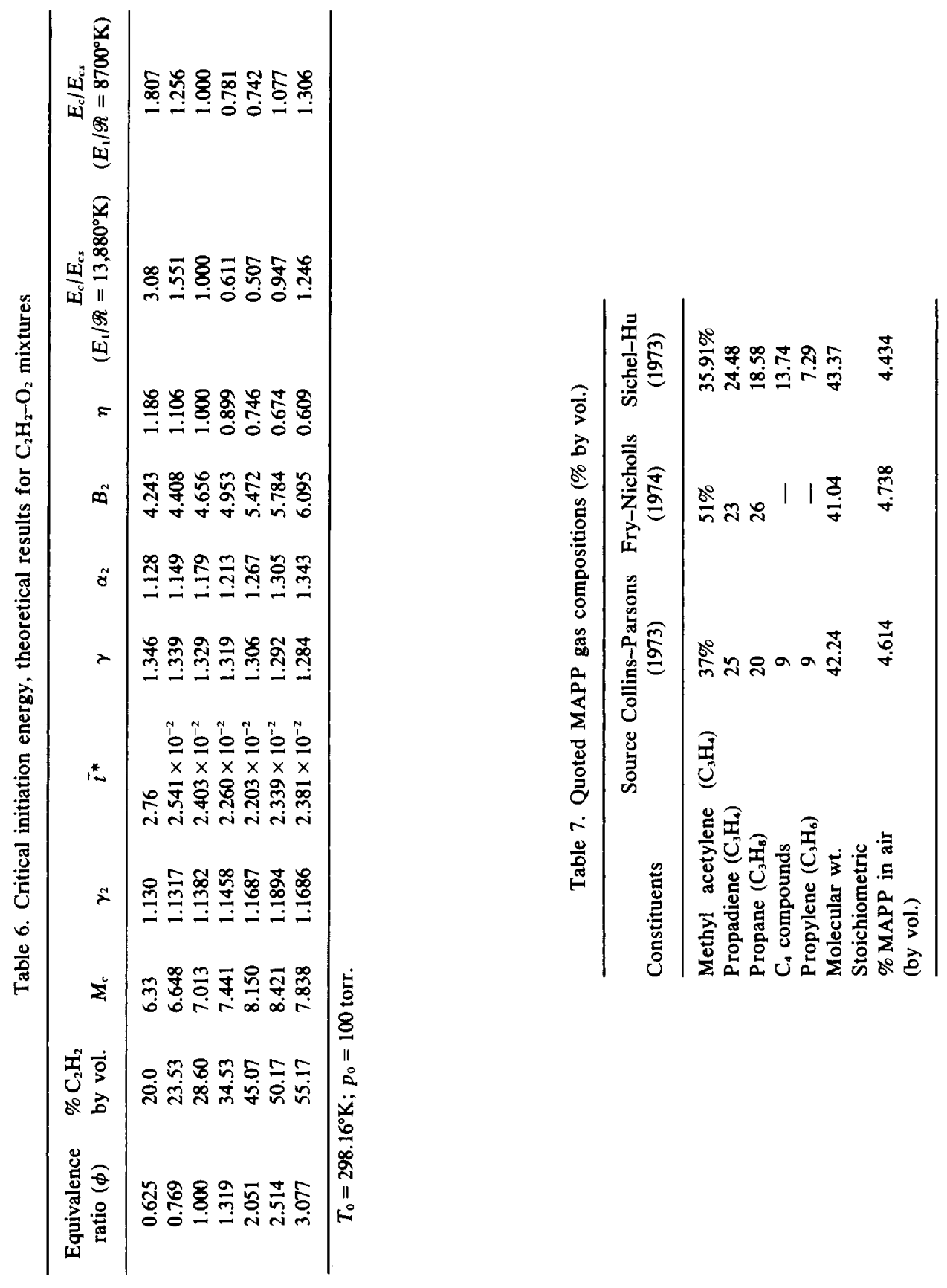


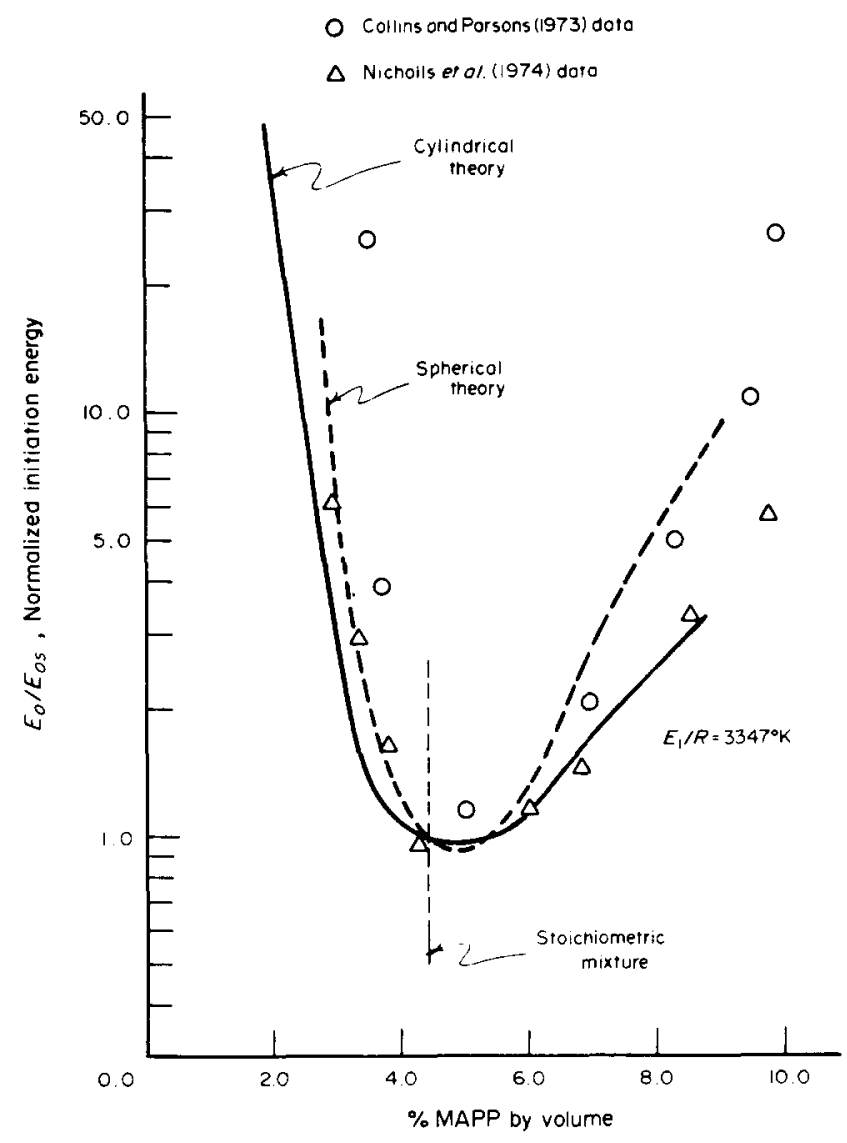

Fig. 1. Experimental and theoretical variations of normalized initiation energy for MAPP-air mixtures.

to the stoichiometric mixture and essentially appears to reproduce the detonation limits. The theory also reproduces the initial drop in initiation energy as the mixture ratio exceeds the stoichiometric value. With $E_{1} / \mathscr{R}=8700^{\circ} \mathrm{K}$ the theoretical value of $E_{c} / E_{c s}$ is below experiment for lean mixtures and is somewhat above the measured value for rich mixtures. With $E_{1} / \mathscr{R}=13,880^{\circ} \mathrm{K}$, the theory produces the correct rich detonation limit but yields low values of $E_{c} / E_{c s}$ between 30 and $50 \% \mathrm{C}_{2} \mathrm{H}_{2}$ by volume. Optimum agreement between theory and experiment would probably be obtained for an activation temperature $E_{1} / \mathscr{R}$ lying between the two values above. What is significant is the essential agreement between the value $E_{1} / \mathscr{R}$ suggested by the initiation theory presented here and the value reported by White (1967).

Equation (23) suggests that $E_{c} \propto \rho_{0}^{1-\nu}$, or for fixed ambient temperature, that $E_{c} \propto p_{0}{ }^{1-\nu}$. Measured initiation energies for $\mathrm{C}_{2} \mathrm{H}_{2}$ at reduced pressures have been reported by Bach et al. (1971), Matsui (1973), Lee and Ramamurthi (1975) and Lee and Matsui (1976). Some of this data which is discussed in detail by Lee 


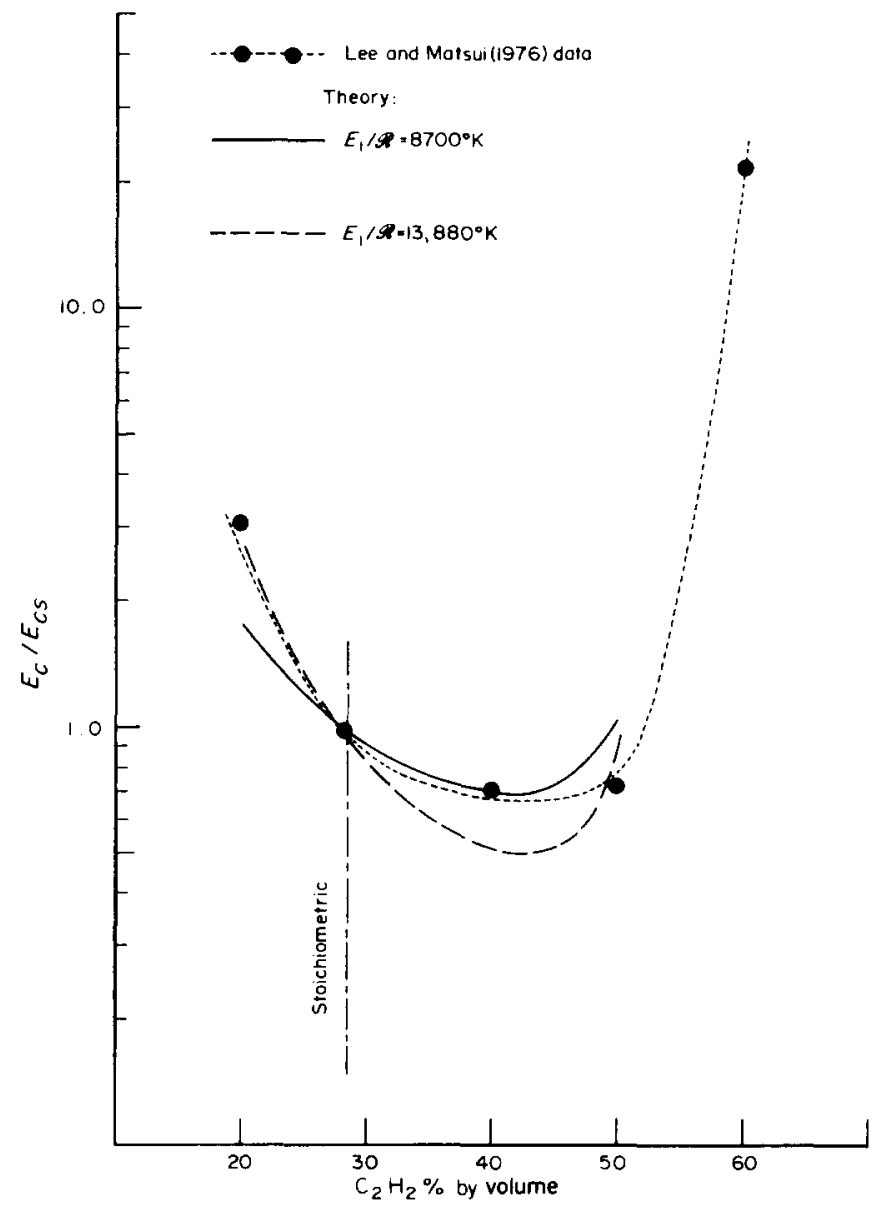

Fig. 2. Experimental and theoretical variation of normalized initiation energy for acetylene-oxygen mixtures.

and Matsui (1976), is plotted in Fig. 3 along with curves corresponding to cylindrical and spherical symmetry with $E_{c} \propto p_{0}{ }^{-1}, p_{0}{ }^{-2}$.

The electric spark initiation experiments reported by Lee and Matsui (1976) were designed to produce waves with cylindrical symmetry and, it can be seen from Fig. 3, that the variation of $E_{c}$ with $p_{0}$ closely follows $E_{c} \propto p_{0}^{-1}$ as predicted by the present theory. The earlier laser spark measurements by Bach et al. (1971) also appear to vary inversely with $p_{0}$, again suggesting cylindrical symmetry. The actual value of $E_{c}$ is very sensitive to the energy source used for initiation, as discussed in detail by Lee and Matsui (1976). The different properties of laser and electric sparks thus result in the difference in the absolute values of $E_{c}$ shown in Fig. 3 .

Matsui (1973) used a planar detonation wave traveling in a tube as the initiation source and determined the minimum tube diameter needed to initiate a detonation in a larger surrounding volume. This method of initiation closely simulates the initiation of a spherical detonation with a point source of energy. 


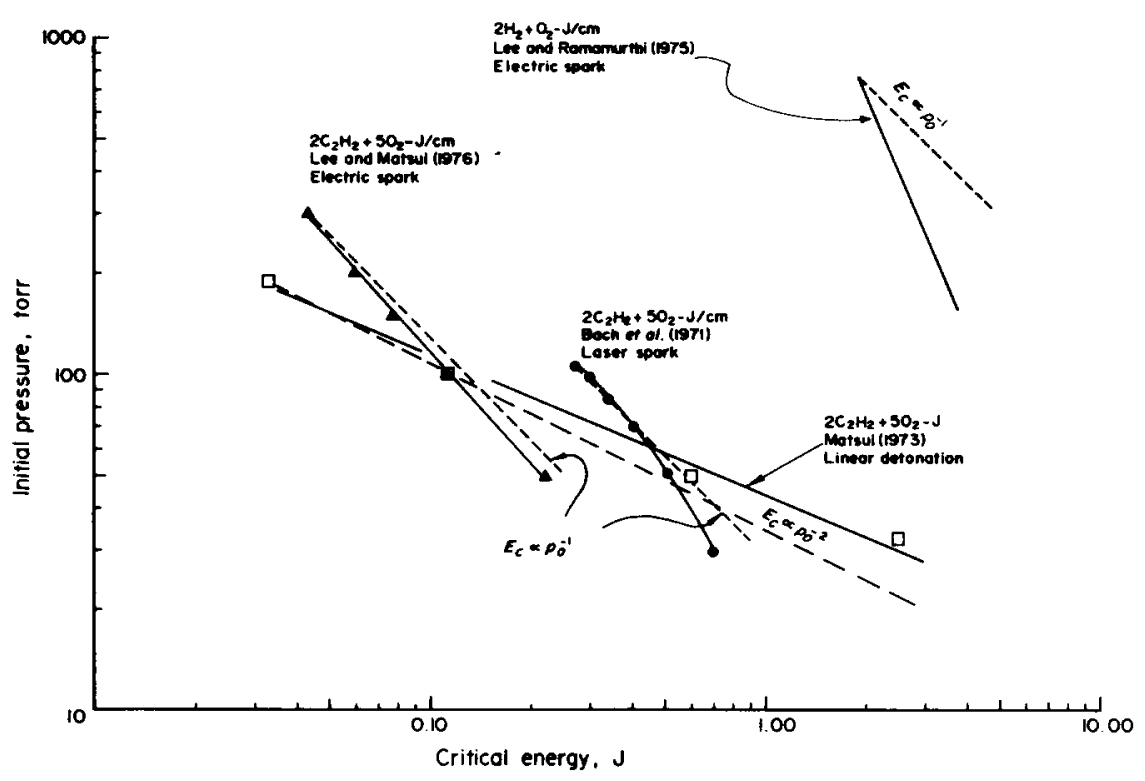

Fig. 3. The variation of critical initiation energy with pressure.

Lee and Matsui (1976) used the critical tube diameter data to estimate the critical initiation energies plotted in Fig. 3. There is close agreement between the Matsui (1973) data and the variation $E_{c} \propto p_{0}{ }^{-2}$ predicted for spherical symmetry by the present theory.

Lee and Ramamurthi (1975) also measured the variation of $E_{c}$ with $p_{0}$ for a stoichiometric hydrogen oxygen mixture. Figure 3 shows that while the $2 \mathrm{H}_{2}-\mathrm{O}_{2}$ initiation energy increases with decreasing $p_{0}$, the rate of increase appears to be much less than indicated by (20), possible because (16) is not a good approximation for $\mathrm{H}_{2}-\mathrm{O}_{2}$ kinetics. An interesting consequence of (23) is that $E_{\mathrm{c}}$ should be independent of $\rho_{0}$ for plane waves.

Bach et al. (1971) expressed their critical initiation energy in terms of the ratio of $\Delta_{c}$, the induction distance of the C-J detonation to the explosion radius $R_{0}$. The modified Zel'dovich criterion presented here can be expressed in the same form by combining eqn (8) with the expressions (14) and (19) for the induction distance $\Delta$ and the ignition delay time $\tau_{1}$ with the result

$$
\frac{\Delta_{c}}{R_{0}} \leqq \delta M_{c} \bar{t}^{*}\left(\frac{\nu+2}{2}\right) \sqrt{ }\left(\frac{\alpha_{\nu}}{k_{\nu}}\right) \exp \frac{E_{1}}{\mathscr{R} T_{0}}\left(\frac{T_{0}}{T_{2 c}}-B_{\nu} \bar{t}^{*} \frac{2 \nu}{\nu+2}\right)
$$

Here $T_{2 c}$ is the temperature downstream of the shock but upstream of the reaction zone for the $\mathrm{C}-\mathrm{J}$ detonation. Bach et al. (1971) computed a critical value of $\left(\Delta_{c} / R_{0}\right)=0.091$ for the initiation of a spherical detonation in a stoichiometric $\mathrm{C}_{2} \mathrm{H}_{2}-\mathrm{O}_{2}$ mixture with $p_{0}=100$ torr. By using (24), this value of $\left(\Delta_{c} / R_{0}\right)$ and the detonation parameters of $\mathrm{C}_{2} \mathrm{H}_{2}-\mathrm{O}_{2}$, it now is possible to determine the corresponding value of the parameter $\delta$. With $M_{c}=7.013, E_{1} / \mathscr{R}=8700^{\circ} \mathrm{K}$, 
$\alpha_{3}=1.0, B_{3}=3.085, \bar{t}^{*}=6.272 \times 10^{-2}, T_{2 c} / T_{0}=8.887$, the result is $\delta=0.282$. This certainly is not an unreasonable value.

\section{Discussion}

The theory developed here verifies that the minimum initiation energy is determined by a balance between the combustion heat release and the growth of the induction zone. The simple relation (22) for the variation of the initiation energy with the fuel-oxidizer mixture ratio, which is developed on the basis of the Zel'dovich criterion, is in excellent agreement with experimental measurements for two quite different, fuel-oxidizer combinations. The theory can be used to compute the initiation energy $E_{c}$ at any fuel-oxidizer ratio, given a measured value of $E_{\mathrm{c}}$ for one particular mixture and an estimate of the activation energy of the induction reaction. The experimentation required to establish the initiation characteristics of a given fuel can thereby be reduced appreciably. With sufficient information about the chemistry of the induction zone, the theory will also predict the effect of ambient density upon the initiation energy.

The simplicity of the theory comes from the use of self-similar strong blast theory to describe the initiating blast. Thus, the theory really is the zeroth order solution of an expansion scheme in which the first order solution would account for the influence of combustion on the initiating blast. The success of this simple theory in the case of explosively and spark initiated detonation waves suggests that non-ideal blast behavior during the initial phases of blast formation will not have a large effect on initiation. At the same time, the critical phenomena which determine whether or not the initiating blast wave quenches appear to occur near $R_{s}=R_{s}^{*}$ where the influence of combustion on wave propagation is still relatively small.

The present theory by no means represents a complete solution of the initiation problem. No explicit means of determining the parameter $\delta$ is provided. A key feature of the present development is that the parameter $\delta$ is not required for the computation of $E_{c} / E_{c s}$. However, an absolute theory for computing $E_{c}$ depends crucially upon $\delta$, and here many problems remain. Determination of $\delta$ will require a detailed consideration of the processes in the induction zone leading to uncoupling of combustion from the blast front. Liñan (1975) has attempted to relate this coupling to the rate of increase of the induction distance in reacting blast waves. In the Bach et al. (1971) and Lee and Ramamurthi (1975) theories, uncoupling occurs whenever the wave Mach number drops below the auto-ignition limit. The work of Strehlow (1968), among others, indicates that transverse waves in the reaction zone of detonation waves may also play an important role in the coupling process. A greater understanding of combustion-shock coupling is needed to determine such parameters as $\delta$ and the auto-ignition Mach number a priori.

Another shortcoming of the present theory is that conditions immediately behind the blast wave are used to compute the induction length $\Delta$. In a more complete theory the variation of the temperature and velocity of a fluid particle as it traverses the induction zone should be taken into account. 
The rate of energy release at the blast source can have a large influence on the initiation energy if the rate falls below a certain minimum value. This problem has been studied extensively by Bach et al. (1971), Knystautas and Lee (1975) and Matsui and Lee (1975) using spark initiators. The effect of energy release rate has been ignored in the present theory; hence, this theory will only be valid for initiation sources with high rates of energy deposition.

The theory developed here appears to take the important physical parameters involved in initiation into account, and provides a simple means of estimating minimum initiation energies.

Acknowledgements - This work was supported by the Air Force Armaments Laboratory, Eglin Air Force Base, Florida, under contract F08635-74-C-0123. The many useful suggestions of Prof. J. A. Nicholls, the project director, are also gratefully acknowledged.

The author would also like to thank Prof. J. H. Lee of McGill University who provided the $\mathrm{C}_{2} \mathrm{H}_{2}-\mathrm{O}_{2}$ data and pointed out the connection between the initiation criterion presented here and the original Zel'dovich criterion.

\section{References}

Bach, G. G., Knystautas, R. and Lee, J. H. (1971) Initiation criteria for diverging gaseous detonations. In Thirteenth Symposium (International) on Combustion, p. 1097. The Combustion Institute, Pittsburgh, Penn.

Collins, P. M. and Parsons, G. H. (1973) Detonation initiation in unconfined fuel-air mixtures. In Proceedings of the Conference on Mechanisms of Explosion and Blast Waves. Joint Technical Coordinating Group for Air Launched Non-Nuclear Ordnance Working Party for Explosives, Naval Weapons Station, Yorktown, Virginia.

Fry, R. S. and Nicholls, J. A. (1974) Blast initiation and propagation of cylindrical detonations in MAPP-air mixtures. AIAA J. 12, 1703.

Gordon, S. and McBride, B. (1971) Computer program for calculation of complex chemical equilibrium compositions, rocket performance, incident and reflected shocks, and ChapmanJouquet detonation. NASA SP-273.

Knystautas, R. and Lee, J. H. (1976) On the effective energy for direct initiation of gaseous detonations. Comb. and Flame, 27, 221.

Korobeinikov, V. P. (1969) The problem of point explosion in a detonating gas. Astro. Acta 14, 411.

Korobeinikov, B. P., Levin, V. A., Markov, V. V. and Chernyi, G. G. (1972) Propagation of blast waves in a combustible gas. Astro. Acta 17, 529.

Lee, J. H. and Matsui, H. (1977) A comparison of the critical energies for direct initiation of spherical detonations in acetylene-oxygen mixtures. Comb. and Flame. (To appear).

Lee, J. H. and Ramamurthi, K. (1976) On the concept of the critical size of a detonation kernel. Comb. and Flame 27, 331.

Levin, V. A. (1967) The approximate solution of the problem of strong point explosion in combustible mixture. Mekhan, Zhidk. Gasa 1, 122-124.

Liñan, A. (1975) Theoretical modeling of the structure of the flow behind blast waves propagating in unconfined reactive mixtures. Paper presented at 1975 AFOSR Contractor's Meeting on FuelAir Explosion Research, Eglin AFB, Florida.

Matsui, H. and Lee, J. H. (1976) Influence of electrode geometry and spacing on the critical energy for direct initiation of spherical gaseous detonation. Comb. and Flame 27, 217.

Nicholls, J. A., Sichel, M., Fry, R. S., Hu, C., Glass, D. R., DeSaro, K. and Kearney, K. (1973) Fundamental aspects of unconfined explosions. Technical Report AFATL-TR-73-125, Air Force Armament Laboratory, Air Force Systems Command, U.S. Air Force, Eglin AFB, Florida.

Nicholls, J. A., Pierce, G. H., Dye, L., Fry, R., Kearney, K. and Liou, M. (1974a) Mechanism in the detonation regime. Final Report UM-012118-F, Dept. of Aerospace Engrg., The Univ. of Michigan for Fast Reaction Group, Picatinny Arsenal. 
Nicholls, J. A., Sichel, M., Fry, R. S., Hu, C., DeSaro, R. and Kearney, K. (1974b) Fundamental aspects of unconfined explosions. Tech. Rept. AFATL-TR-74-123, Air Force Armament Laboratory, Air Force Systems Command, U.S. Air Force, Eglin AFB, Florida.

Schott, G. O. and Kinsey, J. H. (1958) Kinetic studies of hydroxyl radicals in shock waves-II. Induction times in the hydrogen-oxygen reaction. J. Chem. Phys. 29, 1177.

Sedov, L. I. (1959) Similarity and Dimensional Methods in Mechanics, 4th Edn. Academic Press, New York.

Sichel, M. and Hu, Cheng (1973) The impuse generated by blast waves propagating through combustible mixtures. In Proceedings of the Conference on Mechanisms of Explosion and Blast Waves, Naval Weapons Station, Yorktown, Virginia.

Strehlow, R. A. (1968) Gas phase detonations: Recent developments. Comb. and Flame 12, 81.

Taylor, G. I. (1950) The formation of a blast wave by a very intense explosion Proc. Roy. Soc. A201, $159,175$.

Walker, F. E. and Wasley, R. J. (1975) A general model for the shock initiation of explosives. Rept. UCRL-75722 Rev. 1. Lawrence Livermore Lab., Univ. of Calif.

White, D. R. (1967) In Eleventh Symposium (International) on Combustion, p. 147. The Combustion Institute, Pittsburgh, Penn.

Zel'dovich, Y. B., Kogarko, S. M. and Simonov, N. N. (1956) An experimental investigation of spherical detonation of gases. Sov. Phys. Tech. Phys. 1, 1698. 